\title{
PERIODIC SOLUTIONS AND THEIR STABILITY FOR SOME PERTURBED HAMILTONIAN SYSTEMS
}

\author{
JUAN L.G. GUIRAO ${ }^{1}$, JAUME LLIBRE ${ }^{2}$ JUAN A. VERA ${ }^{3}$ AND BRUCE A. WADE ${ }^{4}$
}

\begin{abstract}
We deal with non-autonomous Hamiltonian systems of one degree of freedom. For such differential systems we compute analytically some of their periodic solutions, together with their type of stability. The tool for proving these results is the averaging theory of dynamical systems. We present some applications of these results.
\end{abstract}

\section{INTRODUCTION AND MAIN RESULTS}

We consider the following perturbed first order differential systems

$$
\frac{d p}{d t}=-\frac{\partial \mathcal{H}}{\partial q}+\varepsilon \mathcal{P}_{1}(q, p, t), \quad \frac{d q}{d t}=\frac{\partial \mathcal{H}}{\partial p}+\varepsilon \mathcal{P}_{2}(q, p, t),
$$

where $\mathcal{H}=\mathcal{H}(q, p)$ is a Hamiltonian function defined in an open set $U$ of $\mathbb{R}^{2}, q$ is the position, $p$ its associated momentum, the functions $\mathcal{P}_{i}$ defined in $U \times \mathbb{R}$ are smooth $2 \pi$ - periodic in $t$, and $\varepsilon$ is a small parameter.

All the lemmas, theorems and corollaries stated in this section are proved in the next two sections. The theorems are proved using the averaging theory, see in the appendix a summary of the results on this theory that we need for proving our theorems. For computing analytically periodic solutions of the differential equations we shall use the averaging theory, see for instance $[2,3,4]$, but in those papers we studied periodic solutions of autonomous Hamiltonian systems, and in the present one we are working with nonautonomous differential systems.

We assume that the Hamiltonian $\mathcal{H}$ is expressed in action-angle variables $(I, \theta)$ as $\mathcal{H}(p, q)=\mathcal{H}_{0}(I)$. If the change of variable $(p, q) \mapsto(I, \theta)$ is given by $I=I(p, q)$ and $\theta=\theta(p, q)$, then we consider the functions

$$
\begin{aligned}
& \mathcal{F}_{1}(I, \theta, t)=\frac{\partial I}{\partial p} \mathcal{P}_{1}(q, p, t)+\frac{\partial I}{\partial q} \mathcal{P}_{2}(q, p, t), \\
& \mathcal{F}_{2}(I, \theta, t)=\frac{\partial \theta}{\partial p} \mathcal{P}_{1}(q, p, t)+\frac{\partial \theta}{\partial q} \mathcal{P}_{2}(q, p, t) .
\end{aligned}
$$

The following result holds taking into account that the change of variables to action-angle variables is canonical.

1991 Mathematics Subject Classification. [2010]Primary 34C10, 34C25.

Key words and phrases. Hamiltonian system, periodic solutions, averaging theory. 
Lemma 1. System (1) in action-angle variables $(I, \theta)$ becomes

$$
\frac{d I}{d t}=\varepsilon \mathcal{F}_{1}(I, \theta, t), \quad \frac{d \theta}{d t}=\omega(I)+\varepsilon \mathcal{F}_{2}(I, \theta, t),
$$

where $\omega(I)=d \mathcal{H}_{0}(I) / d I$.

The next result provides sufficient conditions for the existence and the stability of $2 \pi$-periodic solutions of system (2) when the functions $\mathcal{P}_{i}$ 's are independent of $t$, and consequently the functions $\mathcal{F}_{i}$ 's are also independent of $t$.

Theorem 2. If the functions $\mathcal{F}_{i}$ 's do not depend on the time $t$, are $2 \pi-$ periodic in the variable $\theta$, and if $I_{0}$ is a simple zero of the function

$$
\mathcal{F}(I)=\frac{1}{2 \pi} \int_{0}^{2 \pi} \frac{\mathcal{F}_{1}(I, \theta)}{\omega} d \theta,
$$

then there exists a $2 \pi$-periodic solution $I_{\varepsilon}(\theta)$ of system (2) such that $I_{\varepsilon}(0) \rightarrow$ $I_{0}$ when $\varepsilon \rightarrow 0$. Moreover, if $\mathcal{F}^{\prime}\left(I_{0}\right)>0$ then the periodic solution $I_{\varepsilon}(\theta)$ is unstable, and if $\mathcal{F}^{\prime}\left(I_{0}\right)<0$ it is stable.

Assume that the functions $\mathcal{P}_{i}$ are periodic in the variable $t$ with period $T=2 \pi / \omega_{0}$, being $\omega_{0}$ a real positive number. We shall study the existence of $T$ periodic solutions of system (2). We shall consider the Hamiltonian $\mathcal{H}$ linearizable with $\omega(I)=\omega_{0}$ constant. In this case by means of the change of variables $(I, \theta) \longleftrightarrow(I, \varphi)$ given by $I=I$ and $\theta=\varphi+\omega_{0} t$, system (2) is transformed into the system

$$
\frac{d I}{d t}=\varepsilon \mathcal{F}_{1}\left(I, \varphi+\omega_{0} t, t\right), \quad \frac{d \varphi}{d t}=\varepsilon \mathcal{F}_{2}\left(I, \varphi+\omega_{0} t, t\right) .
$$

The following result provides sufficient conditions for the existence and the stability of $T$-periodic solutions of system (3).

Theorem 3. If the perturbed functions $\mathcal{F}_{i}$ are $T$-periodic in the variable $t$ and $\left(I_{0}, \varphi_{0}\right)$ is a zero of the function

$$
\mathfrak{F}(I, \varphi)=\frac{1}{T}\left(\int_{0}^{T} \mathcal{F}_{1}\left(I, \varphi+\omega_{0} t, t\right) d t, \int_{0}^{T} \mathcal{F}_{2}\left(I, \varphi+\omega_{0} t, t\right) d t\right),
$$

and the Jacobian $\operatorname{det}\left(D \mathfrak{F}\left(I_{0}, \varphi_{0}\right)\right) \neq 0$, then there exists a $T$-periodic solution $\gamma_{\varepsilon}(t)$ of system (3) such that $\gamma_{\varepsilon}(0) \rightarrow\left(I_{0}, \varphi_{0}\right)$ when $\varepsilon \rightarrow 0$. Moreover, if one of the two roots of the characteristic polynomial of the Jacobian matrix $D \mathfrak{F}\left(I_{0}, \varphi_{0}\right)$ have positive real part the periodic solution $\gamma_{\varepsilon}(t)$ is unstable.

If the two roots of that characteristic polynomial have negative real part, then the periodic solution $\gamma_{\varepsilon}(t)$ is stable.

Now we are going to present some applications of the previous theorems. 
Using Theorem 2 we will study the periodic solutions of the following second-order differential equation

$$
\frac{d^{2} x}{d t^{2}}+\frac{k}{x^{2}}=\varepsilon \mathcal{P}\left(x, \frac{d x}{d t}\right),
$$

where $k \in \mathbb{R}^{+}$and $\varepsilon$ is a small parameter. Note that when $\varepsilon=0$ we have the 1-dimensional Kepler problem, see for instance [6] and [7].

As we shall see in subsection 3.1 doing the change of time $t \mapsto E$ given by $t=(E-\sin E) / \omega$, the elliptic collision solutions of the 1-dimensional Kepler problem become periodic solutions. We recall that the variable $E$ is named as the eccentric anomaly. We want to study which of these periodic solutions persist under the perturbation given in (4).

Theorem 4. If $I_{0}$ is a simple zero of the function

$$
\mathcal{F}(I)=\frac{1}{2 \pi} \int_{0}^{2 \pi} \frac{k}{2 \omega^{2} I} \sin E \mathcal{P}\left(\frac{I^{2}}{k}(1-\cos E), \frac{k \sin E}{2 I(1-\cos E)}\right) d E,
$$

then it exists a $2 \pi$-periodic solution $x_{\varepsilon}(E)$ of the differential equation (4) such that $x_{\varepsilon}(0) \rightarrow I_{0}$ when $\varepsilon \rightarrow 0$. Moreover, if $\mathcal{F}^{\prime}\left(I_{0}\right)>0$ then the periodic solution $x_{\varepsilon}(E)$ is unstable, and if $\mathcal{F}^{\prime}\left(I_{0}\right)<0$ it is stable.

See a numerical example of the periodic solution of Theorem 4 after the proof of this theorem.

Corollary 5. For $\varepsilon \neq 0$ sufficiently small the differential equation

$$
\frac{d^{2} x}{d t^{2}}+\frac{k}{x^{2}}=\varepsilon\left(a+b x^{n}\right) \frac{d x}{d t},
$$

where $n \in \mathbb{N}$ and $a, b \in \mathbb{R}$, has a periodic solution if $a b<0$.

Corollary 6. For $\varepsilon \neq 0$ sufficiently small and for any positive integer $N$ the differential equation

$$
\frac{d^{2} x}{d t^{2}}+\frac{k}{x^{2}}=\varepsilon \sin \left(x \frac{d x}{d t}\right)
$$

has at least $N$ periodic solutions.

Using Theorem 3 we will study the periodic solutions of the second-order differential equation

$$
\frac{d^{2} x}{d t^{2}}+\omega_{0}^{2} x=\varepsilon x^{2}\left(a+b x^{2}\right) \sin \left(\omega_{0} t\right)
$$

with $\omega_{0}>0$ and $a b>0$.

Corollary 7. The differential equation (5) has at least four periodic solutions

$$
x_{i}(t, \varepsilon)=\sqrt{\frac{2 I_{i}}{\omega_{0}}} \cos \left(\varphi_{i}+\omega_{0} t\right)+O(\varepsilon),
$$


for $i=1,2,3,4$, with $\left(I_{1}, \varphi_{1}\right)=\left(a \omega_{0} / b, 0\right),\left(I_{2}, \varphi_{2}\right)=\left(a \omega_{0} / b, \pi\right),\left(I_{3}, \varphi_{3}\right)=$ $\left(2 a \omega_{0} /(3 b), \pi / 2\right),\left(I_{4}, \varphi_{4}\right)=\left(2 a \omega_{0} /(3 b), \pi\right)$. The periodic solution $x_{1}$ is stable and the periodic solutions $x_{i}$ for $i=2,3,4$ are unstable.

A numerical application of Corollary 7 and consequently of Theorem 3 appears after the proof of Corollary 7 .

\section{Proofs of our MAIN RESUlts}

Proof Lemma 1. The unperturbed system of system (1)

$$
\frac{d p}{d t}=-\frac{\partial \mathcal{H}}{\partial q}, \quad \frac{d q}{d t}=\frac{\partial \mathcal{H}}{\partial p},
$$

has a very simple formulation in action-angle variables, namely

$$
\frac{d I}{d t}=0, \quad \frac{d \theta}{d t}=\omega .
$$

Since the Poisson Bracket of two smooth functions $f$ and $g$ is defined as

$$
\{f, g\}=\frac{\partial f}{\partial q} \frac{\partial g}{\partial p}-\frac{\partial f}{\partial p} \frac{\partial g}{\partial q},
$$

we have that

and

$$
\frac{d I}{d t}=\frac{\partial I}{\partial q} \frac{\partial \mathcal{H}}{\partial p}-\frac{\partial I}{\partial p} \frac{\partial \mathcal{H}}{\partial q}=\{I, \mathcal{H}\}=0
$$

$$
\frac{d \theta}{d t}=\frac{\partial \theta}{\partial q} \frac{\partial \mathcal{H}}{\partial p}-\frac{\partial \theta}{\partial p} \frac{\partial \mathcal{H}}{\partial q}=\{\theta, \mathcal{H}\}=\omega
$$

The variation with the time of the action $I$ of the perturbed system (1) satisfies

$$
\begin{aligned}
\frac{d I}{d t} & =\frac{\partial I}{\partial p} \frac{d p}{d t}+\frac{\partial I}{\partial q} \frac{d q}{d t} \\
& =\frac{\partial I}{\partial p}\left(-\frac{\partial \mathcal{H}}{\partial q}+\varepsilon P_{1}(q, p)\right)+\frac{\partial I}{\partial q}\left(\frac{\partial \mathcal{H}}{\partial p}+\varepsilon P_{2}(q, p)\right) \\
& =\varepsilon\left(\frac{\partial I}{\partial p} P_{1}(q, p)+\frac{\partial I}{\partial q} P_{2}(q, p)\right) .
\end{aligned}
$$

The variation with the time of the angle $\theta$ satisfies

$$
\begin{aligned}
\frac{d \theta}{d t} & =\frac{\partial \theta}{\partial p} \frac{d p}{d t}+\frac{\partial \theta}{\partial q} \frac{d q}{d t} \\
& =\frac{\partial \theta}{\partial p}\left(-\frac{\partial \mathcal{H}}{\partial q}+\varepsilon P_{1}(q, p)\right)+\frac{\partial \theta}{\partial q}\left(\frac{\partial \mathcal{H}}{\partial p}+\varepsilon P_{2}(q, p)\right) \\
& =\omega+\varepsilon\left(\frac{\partial \theta}{\partial p} P_{1}(q, p)+\frac{\partial \theta}{\partial q} P_{2}(q, p)\right) .
\end{aligned}
$$


Then the differential system (1) is transformed into the differential system (2).

Proof Theorem 2. If the functions $\mathcal{F}_{i}$ are independent of the time $t$, then system (2) is autonomous. Taking as new independent variable the variable $\theta$ system (2) becomes the differential equation

$$
\frac{d I}{d \theta}=\frac{\varepsilon \mathcal{F}_{1}(I, \theta)}{\omega+\varepsilon \mathcal{F}_{2}(I, \theta)}=\varepsilon \frac{\mathcal{F}_{1}(I, \theta)}{\omega}+O\left(\varepsilon^{2}\right)
$$

Now using the first order averaging theory (see Theorem 8 of the appendix) we obtain the averaged function

$$
\frac{1}{2 \pi} \int_{0}^{2 \pi} \frac{\mathcal{F}_{1}(I, \theta)}{\omega} d \theta
$$

and the statements of the theorem follows.

Proof Theorem 3. Applying again the first order averaging theory but now to the differential system (3) we obtain the averaged function $\mathfrak{F}(\rho, \varphi)$. Then the statement of Theorem 3 follows directly from the statement of Theorem 8 of the appendix.

\section{Applications of our main Results}

As an application of Theorems 2 and 3 we will study the following problems.

\subsection{Periodic solutions of the perturbed 1-dimensional Kepler Prob-} lem.

Proof of Theorem 4. Doing the change of variables $x=q$ and $\frac{d x}{d t}=p$, the second order differential equation (4) becomes the first order differential system

$$
\frac{d p}{d t}=-\frac{k}{q^{2}}+\varepsilon \mathcal{P}(q, p), \quad \frac{d q}{d t}=p
$$

We compute the action-angle variables for the Hamiltonian $\mathcal{H}: \mathbb{R} \times(0,+\infty) \rightarrow \mathbb{R}$ given by $\mathcal{H}(p, q)=\frac{p^{2}}{2}-\frac{k}{q}$. The action is given by $I=\frac{1}{2 \pi} \oint p d q$ computed in a closed curve of the phase space at the energy level $\mathcal{H}=h<0$. Then

$$
I=\frac{1}{\pi} \lim _{s \rightarrow 0} \int_{s}^{-k / h} \sqrt{\frac{2 k}{q}+2 h} d q=\frac{k}{2 \sqrt{-h}} .
$$


Solving the equation $I=\frac{k}{2 \sqrt{-h}}$ we obtain $h=-\frac{k^{2}}{4 I^{2}}$. Then $\mathcal{H}(p, q)=$ $-\frac{k^{2}}{4 I^{2}}=\mathcal{H}_{0}(I)$. We consider the generating function

$$
\mathcal{W}(q, I)= \pm \int_{q}^{4 I^{2} / k} \sqrt{\frac{2 k}{s}-\frac{k^{2}}{2 I^{2}}} d s
$$

Then we obtain

$$
p=\frac{\partial \mathcal{W}}{\partial q}=\mp \sqrt{\frac{2 k}{q}-\frac{k^{2}}{2 I^{2}}}, \quad \theta=\frac{\partial \mathcal{W}}{\partial I}=\frac{\partial}{\partial I}\left( \pm \int_{q}^{4 I^{2} / k} \sqrt{\frac{2 k}{s}-\frac{k^{2}}{2 I^{2}}} d s\right) .
$$

Since $\frac{d \theta}{d t}=\omega=\frac{d \mathcal{H}_{0}}{d I}=\frac{k^{2}}{2 I^{3}}$, we get $\theta=\frac{k^{2}}{2 I^{3}} t=\omega t$.

Fixed the energy level $h$, from $\frac{p^{2}}{2}-\frac{k}{q}=h$ and using $p=\frac{d q}{d t}$, we obtain $\frac{d q}{d t}= \pm \sqrt{\frac{2 k}{q}+2 h}$ and $d t= \pm \frac{d q}{\sqrt{\frac{2 k}{q}+2 h}}$. From the relation $h=-\frac{k^{2}}{4 I^{2}}$, we obtain

$$
t= \pm \int_{q}^{4 I^{2} / k} \frac{1}{\sqrt{\frac{2 k}{s}-\frac{k^{2}}{2 I^{2}}}} d s .
$$

Note that the $t$ in this last equality is the time that needs the particle for going from the position $q$ to the maximal value of its position obtained solving the equation

$$
\frac{d q}{d t}=\sqrt{\frac{2 k}{q}+h}=0
$$

Doing the change of variable $q=\frac{I^{2}}{k}(1-\cos E)$ in (7) we obtain

$$
t=\frac{2 I^{3}}{k^{2}}(E-\sin E)=\frac{1}{\omega}(E-\sin E),
$$

and consequently $\theta=E-\sin E$.

Since $-\frac{k^{2}}{4 I^{2}}=\frac{p^{2}}{2}-\frac{k}{q}$, we have

$$
\frac{\partial}{\partial p}\left(-\frac{k^{2}}{4 I^{2}}\right)=\frac{k^{2}}{2 I^{3}} \frac{\partial I}{\partial p}=\frac{\partial}{\partial p}\left(\frac{p^{2}}{2}-\frac{k}{q}\right)=p,
$$

therefore $p=\frac{k^{2}}{2 I^{3}} \frac{\partial I}{\partial p}$. 
Using $\omega=\frac{k^{2}}{2 I^{3}}$ we obtain $\frac{\partial I}{\partial p}=\frac{p}{\omega}$. By the chain rule and the equation of motion we get

Then

$$
\omega=\frac{d \theta}{d t}=\frac{d \theta}{d p} \frac{d p}{d t}=\frac{d \theta}{d p}\left(-\frac{k}{q^{2}}\right) .
$$

$$
\frac{d \theta}{d p}=-\frac{\omega}{k} q^{2}=-\frac{I}{2 k}(1-\cos E)^{2}
$$

Finally, from

$$
q=\frac{I^{2}}{k}(1-\cos E), \quad p=\frac{k}{2 I} \frac{\sin E}{1-\cos E} \quad \text { and } \quad \frac{\partial I}{\partial p}=\frac{p}{\omega},
$$

we obtain

$$
\begin{aligned}
\mathcal{F}_{1}(I, \theta, t) & =\frac{\partial I}{\partial p} \mathcal{P}(q, p) \\
& =\frac{k}{2 \omega I} \frac{\sin E}{1-\cos E} \mathcal{P}\left(\frac{I^{2}}{k}(1-\cos E), \frac{k \sin E}{2 I(1-\cos E)}\right) \\
& =\mathcal{G}_{1}(I, E),
\end{aligned}
$$

and using

we have

$$
\frac{\partial \theta}{\partial p}=\frac{I}{k}(1-\cos E)^{2}
$$

$$
\begin{aligned}
\mathcal{F}_{2}(I, \theta) & =\frac{\partial \theta}{\partial p} \mathcal{P}(q, p) \\
& =\frac{I}{k}(1-\cos E)^{2} \mathcal{P}\left(\frac{I^{2}}{k}(1-\cos E), \frac{k \sin E}{2 I(1-\cos E)}\right) \\
& =\mathcal{G}_{2}(I, E) .
\end{aligned}
$$

Then from Theorem 2 and since $d \theta / d E=1-\cos E$, we obtain

$$
\frac{d I}{d E}=\varepsilon \frac{k}{2 \omega^{2} I} \sin E \mathcal{P}\left(\frac{I^{2}}{k}(1-\cos E), \frac{k \sin E}{2 I(1-\cos E)}\right)+O\left(\varepsilon^{2}\right) .
$$

Again from Theorem 2 it follows the statement of Theorem 4.

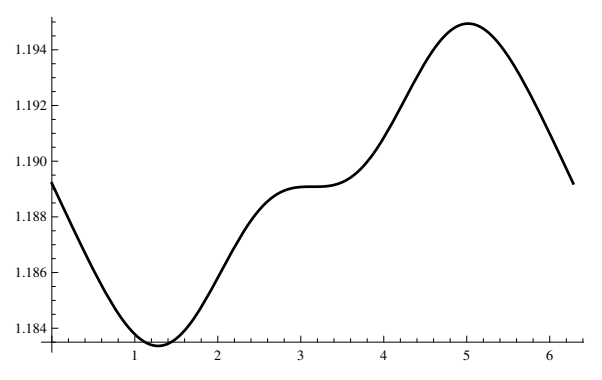

Figure 1

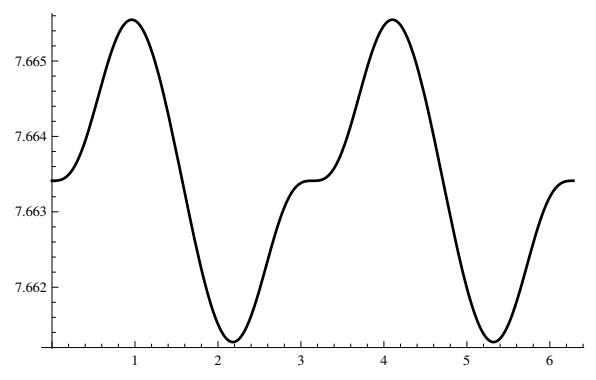

Figure 2 
The two periodic solutions of the 1-D Kepler differential equation with $\mathcal{P}(x, \dot{x})=\left(x^{2}-1\right) \dot{x}$ and $\mathcal{P}(x, \dot{x})=\sin (x \dot{x})$. In the first figure $k=1$, $\varepsilon=1 / 100$ and $I(0)=2^{1 / 4}$. In the second figure $k=1, \varepsilon=10^{-6}$ and $I(0)=7.663411940415026$ the first zero of the Bessel function of first kind. The horizontal axis there is the eccentric anomaly $E$ varying from 0 to $2 \pi$, and the vertical axis the action $I(E)$.

3.2. Periodic solutions of the differential equation $\frac{d^{2} x}{d t^{2}}+\frac{k}{x^{2}}=\varepsilon(a+$ $\left.b x^{n}\right) \frac{d x}{d t}$.

Proof of Corollary 5. From the statement of Theorem 4 and since $\mathcal{P}(q, p)=$ $\left(a+b q^{n}\right) p$ we have

$$
\begin{aligned}
\mathcal{F}(I) & =\frac{1}{2 \pi} \int_{0}^{2 \pi} \frac{k}{2 \omega^{2} I} \sin E \mathcal{P}\left(\frac{I^{2}}{k}(1-\cos E), \frac{k \sin E}{2 I(1-\cos E)}\right) d E \\
& =\frac{1}{2 \pi} \int_{0}^{2 \pi} \frac{k^{2} \sin ^{2} E}{4 \omega^{2} I^{2}(1-\cos E)}\left(a+\frac{b I^{2 n}(1-\cos E)^{n}}{k^{n}}\right) d E \\
& =\frac{1}{2 \pi} \int_{0}^{2 \pi} \frac{I^{4} \sin ^{2} E}{k^{2}(1-\cos E)}\left(a+\frac{b I^{2 n}(1-\cos E)^{n}}{k^{n}}\right) d E,
\end{aligned}
$$

because $\omega=k^{2} /\left(2 I^{3}\right)$. Therefore

$$
\mathcal{F}(I)=\frac{I^{4}}{k^{2}}\left(a+\frac{2^{n} b I^{2 n} \Gamma\left(n+\frac{1}{2}\right)}{\sqrt{\pi} k^{n} \Gamma(n+2)}\right),
$$

where $\Gamma(z)$ is the Euler gamma function, see for more details [1].

The function $\mathcal{F}(I)$ has the unique positive zero

$$
I_{0}=\pi^{\frac{1}{4 n}}\left(-\frac{a k^{n} \Gamma(n+2)}{2^{n} b \Gamma\left(n+\frac{1}{2}\right)}\right)^{\frac{1}{2 n}} .
$$

if $a b<0$. So, from Theorem 4 the corollary follows.

3.3. Periodic solutions of the differential equation $\frac{d^{2} x}{d t^{2}}+\frac{k}{x^{2}}=\varepsilon \sin \left(x \frac{d x}{d t}\right)$.

Proof of Corollary 6. From the statement of Theorem 4 and since $\mathcal{P}(q, p)=$ $\sin (q p)$ we have

$$
\begin{aligned}
\mathcal{F}(I) & =\frac{1}{2 \pi} \int_{0}^{2 \pi} \frac{k}{2 \omega^{2} I} \sin E \mathcal{P}\left(\frac{I^{2}}{k}(1-\cos E), \frac{k \sin E}{2 I(1-\cos E)}\right) d E \\
& =\frac{1}{2 \pi} \int_{0}^{2 \pi} \frac{2 I^{5}}{k^{3}} \sin E \sin \left(\frac{I \sin E}{2}\right) d E,
\end{aligned}
$$


because $\omega=k^{2} /\left(2 I^{3}\right)$. Therefore

$$
\mathcal{F}(I)=\frac{2 I^{5} J_{1}(I / 2)}{k^{3}}
$$

where $J_{1}(z)$ is the Bessel function of first kind. The function $J_{1}(z)$ has infinitely many positive simple zeros accumulating at infinity, see for instance [1]. Hence, from Theorem 4 the corollary follows.

3.4. Periodic solutions of the differential equation $\frac{d^{2} x}{d t^{2}}+\omega_{0}^{2} x=$ $\varepsilon x^{2}\left(a+b x^{2}\right) \sin \left(\omega_{0} t\right)$.

Proof of Corollary \%. The corresponding first order differential system of the second-order differential equation of the statement of this corollary is

$$
\frac{d p}{d t}=-\omega_{0}^{2} q+\varepsilon q^{2}\left(a-b q^{2}\right) \sin \left(\omega_{0} t\right), \quad \frac{d q}{d t}=p .
$$

The Hamiltonian $\mathcal{H}(p, q)=\frac{p^{2}+\omega_{0}^{2} q^{2}}{2}$ has the action-angle variables $(I, \theta)$ given by $p=\sqrt{2 \omega_{0} I} \sin \theta$ and $q=\sqrt{\frac{2 I}{\omega_{0}}} \cos \theta$, see [5] for details on the computation of these action-angle variables.

The Hamiltonian $\mathcal{H}$ in the action-angle variables is $\mathcal{H}(I)=\omega_{0} I$. Computing $\mathcal{F}_{1}$ and $\mathcal{F}_{2}$ we obtain

$$
\mathcal{F}_{1}(I, \theta, t)=\frac{2 I \sqrt{2 \omega_{0} I}\left(a \omega_{0}-2 b I \cos ^{2} \theta\right) \cos ^{2} \theta \sin \left(\omega_{0} t\right)}{\omega_{0}^{3}}
$$

and

$$
\mathcal{F}_{2}(I, \theta, t)=-\frac{\sqrt{2 \omega_{0} I}\left(a \omega_{0}-2 b I \cos ^{2} \theta\right) \cos \theta \sin \left(\omega_{0} t\right)}{\omega_{0}^{3}} .
$$

The map $\mathfrak{F}$ is given by

$$
\mathfrak{F}(I, \varphi)=\left(\frac{I \sqrt{2 \omega_{0} I}\left(a \omega_{0}-b I\right) \cos \varphi}{4 \omega_{0}^{3}}, \frac{\sqrt{2 \omega_{0} I}\left(2 a \omega_{0}-3 b I\right) \sin \varphi}{4 \omega_{0}^{3}}\right) .
$$

The system $\mathfrak{F}(I, \varphi)=(0,0)$ has the four solutions $\left(I_{1}, \varphi_{1}\right)=\left(a \omega_{0} / b, 0\right)$, $\left(I_{2}, \varphi_{2}\right)=\left(a \omega_{0} / b, \pi\right),\left(I_{3}, \varphi_{3}\right)=\left(2 a \omega_{0} /(3 b), \pi / 2\right),\left(I_{4}, \varphi_{4}\right)=\left(2 a \omega_{0} /(3 b), \pi\right)$ and

$$
\begin{aligned}
& \left.D \mathfrak{F}\right|_{(I, \varphi)=\left(I_{1}, \varphi_{1}\right)}=-\left.D \mathfrak{F}\right|_{(I, \varphi)=\left(I_{2}, \varphi_{2}\right)}=\left(\begin{array}{cc}
\lambda & 0 \\
0 & \lambda
\end{array}\right), \\
& \left.D \mathfrak{F}\right|_{(I, \varphi)=\left(I_{3}, \varphi_{3}\right)}=-\left.D \mathfrak{F}\right|_{(I, \varphi)=\left(I_{4}, \varphi_{4}\right)}=\left(\begin{array}{cc}
0 & \lambda_{1} \\
\lambda_{2} & 0
\end{array}\right),
\end{aligned}
$$

with

$$
\lambda=-\frac{a \sqrt{a}}{2 \sqrt{2 b} \omega_{0}}, \lambda_{1}=-\frac{\sqrt{3} a^{2} \sqrt{a}}{27 b \sqrt{b} \omega_{0}}, \lambda_{2}=-\frac{\sqrt{3 a} b}{2 \omega_{0}^{2} \sqrt{b}} .
$$


The two eigenvalues corresponding to the linearized Poincaré map associated to the periodic orbit $\gamma_{1}$ are $\lambda=-\frac{a \sqrt{a}}{2 \sqrt{2 b} \omega_{0}}$ and $\lambda=-\frac{a \sqrt{a}}{2 \sqrt{2 b} \omega_{0}}$.

The two eigenvalues corresponding to the linearized Poincaré map associated to the periodic orbit $\gamma_{2}$ are $\lambda=\frac{a \sqrt{a}}{2 \sqrt{2 b} \omega_{0}}$ and $\lambda=\frac{a \sqrt{a}}{2 \sqrt{2 b} \omega_{0}}$.

The two eigenvalues corresponding to the linearized Poincaré map associated to the periodic orbit $\gamma_{3}$ and $\gamma_{4}$ are $\pm \sqrt{\lambda_{1} \lambda_{2}}$.

Applying Theorem 8 and the canonical change of variables we obtain immediately the results stated in the corollary.

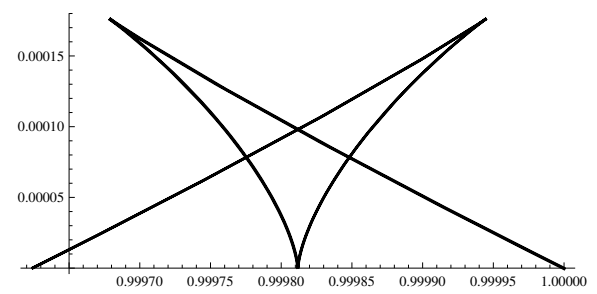

Figure 3

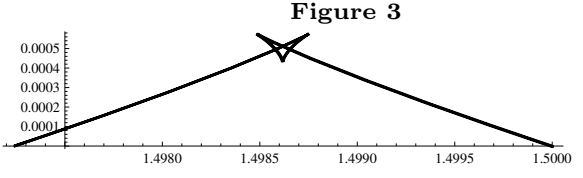

Figure 5

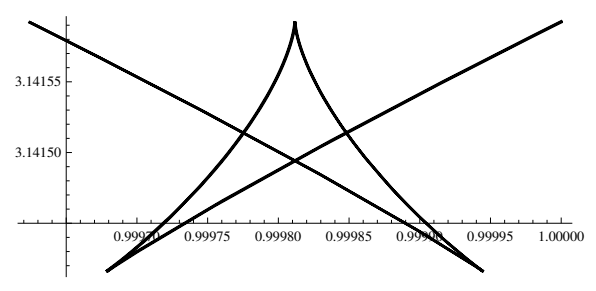

Figure 4

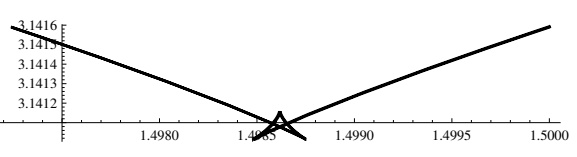

Figure 6

The four periodic solutions of Corollary 7 corresponding to the differential equation (5) with $a=b=\omega=1$ and $\varepsilon=1 / 1000$ are shown in Figures 3, 4, 5 and 6 . In the horizontal axis there is the action $I(t)$ and in the vertical one the angle $\varphi(t)$.

\section{Conclusion Section}

In this paper we consider non-autonomous Hamiltonian systems of one degree of freedom, and we show how to compute analytically some of their periodic solutions, together with their type of stability, using the averaging theory. We illustrate this tool studying two kinds of non-autonomous Hamiltonian systems of one degree of freedom, see Theorems 2 and 3 and its applications Corollaries 5, 6 and 7 .

\section{APPEndix: AverAging theORY OF FIRST ORDER}

We deal with the two initial value problems

$$
\dot{\mathbf{x}}=\varepsilon F_{1}(t, \mathbf{x})+\varepsilon^{2} F_{2}(t, \mathbf{x}, \varepsilon), \quad \mathbf{x}(0)=\mathbf{x}_{0},
$$


and

$$
\dot{\mathbf{y}}=\varepsilon f(\mathbf{y}), \quad \mathbf{y}(0)=\mathbf{x}_{0},
$$

where the variables $\mathbf{x}, \mathbf{y}$ and $\mathbf{x}_{0}$ are in the open set $\Omega \subset \mathbb{R}^{n}, t \in[0, \infty)$ and $\varepsilon \in\left(0, \varepsilon_{0}\right]$. The functions $F_{1}$ and $F_{2}$ which appear in $(8)$ are $T$-periodic in the variable $t$. The averaged function of system (8) is defined by

$$
f(\mathbf{y})=\frac{1}{T} \int_{0}^{T} F_{1}(t, \mathbf{y}) d t .
$$

Theorem 8. Suppose that the functions $F_{1}, D_{\mathbf{x}} F_{1}, D_{\mathbf{x x}} F_{1}, F_{2}$ and $D_{\mathbf{x}} F_{2}$ are continuous, bounded by a constant independent of $\varepsilon$ in $[0, \infty) \times \Omega \times\left(0, \varepsilon_{0}\right]$, and that $y(t) \in \Omega$ for $t \in[0,1 / \varepsilon]$. Then the following statements hold.

(a) $\mathbf{x}(t)-\mathbf{y}(t)=O(\varepsilon)$ as $\varepsilon \rightarrow 0$ for $t \in[0,1 / \varepsilon]$.

(b) If $p \neq 0$ satisfies $f(p)=0$ and the Jacobian $\operatorname{det}\left(D_{\mathbf{y}} f(p)\right) \neq 0$, then there is a T-periodic solution $\mathbf{x}(t, \varepsilon)$ of the differential system (8) such that $\phi(0, \varepsilon)=p+O(\varepsilon)$.

(c) If a real part of some eigenvalue of the Jacobian matrix $D_{\mathbf{y}} f(p)$ is positive, then the periodic solution $\mathbf{x}(t, \varepsilon)$ is unstable. The periodic solution $\mathbf{x}(t, \varepsilon)$ is stable if all the real parts of the eigenvalues of the Jacobian matrix $D_{\mathbf{y}} f(p)$ are negative.

For a proof of Theorem 8 see for instance Chapter 11 of [8].

\section{ACKNOWLEDGEMENTS}

The first author is partially supported by Ministerio de Ciencia, Innovación y Universidades grant number PGC2018-097198-B-I00 and Fundación Séneca de la Región de Murcia grant number 20783/PI/18.

The second author is partially supported by the Ministerio de Ciencia, Innovación y Universidades, Agencia Estatal de Investigación grant MTM2016-77278-P (FEDER), the Agència de Gestió d'Ajuts Universitaris i de Recerca grant 2017SGR1617, and the H2020 European Research Council grant MSCA-RISE-2017-777911.

\section{REFERENCES}

[1] M. Abramowitz And I. A. Stegun, Handbook of mathematical functions, National Bureau of Standards Applied Mathematics Series Vol. 55, Tenth Printing, Washington, 1972.

[2] M. Kulesza And J. LliBre, The 3-dimensional cored and logarithm potentials: periodic orbits, J. Math. Physics 55 (2014), 112702-pp 10.

[3] F.E. Lembarki and J. Llibre, Periodic orbits for the generalized Yang-Mills Hamiltonian system in dimension 6, Nonlinear Dynamics 76 (2014), 1807-1819.

[4] J. Llibre And C. Vidal, New 1: $1: 1$ periodic solutions in 3-dimensional galactictype Hamiltonian systems, Nonlinear Dynamics 78 (2014), 969-980, 
[5] K.R. Meyer, G.R. Hall And D. Offin, Introduction to Hamiltonian Dynamical Systems and the N-Body Problem, Applied Mathematical Sciences 90, Second Edition, Springer-Verlag, 2009.

[6] A.E. Roy, Orbital motion, Halsted Press,John Wiley \& Sons, New York, 1978.

[7] E. L. Stiefel And G. Scheifele. Linear and Regular Celestial Mechanics. SpringerVerlag Berlin Heidelberg. 1971.

[8] F. Verhulst, Nonlinear Differential Equations and Dynamical Systems, Universitext, Springer, New York, 1996.

${ }^{1}$ Departamento de Matemáca Aplicada y Estadística. Universidad Politécnica de Cartagena, 30202-Cartagena, Región de Murcia, Spain, Spain

Email address: juan.garcia@upct.es

${ }^{1}$ Departament de Matemàtiques. Universitat Autònoma de Barcelona, Bellaterra, 08193-Barcelona, Catalonia, Spain

Email address: jllibre@mat.uab.cat

3 Centro Universitario de la Defensa. Academia General del Aire. Universidad Politécnica de Cartagena, 30720-Santiago de la Ribera, Región de Murcia, Spain

Email address: juanantonio.vera@upct.es

4 Mathematics Department, P.O. Box 43568, University of Louisiana at LAFAYETTE, LAFAYETTE, LA 70504-3568, USA

Email address: bwade@louisiana.edu 